\title{
Sleep duration and the risk of cancer: a systematic review and meta-analysis including dose-response relationship
}

Yuheng Chen ${ }^{1 \dagger}$, Fengwei Tan $^{2 \dagger}$, Luopei Wei ${ }^{3}$, Xin Li ${ }^{3}$, Zhangyan Lyu ${ }^{3}$, Xiaoshuang Feng ${ }^{3}$, Yan Wen ${ }^{3}$, Lanwei Guo ${ }^{3,4}$, Jie $\mathrm{He}^{2}$, Min $\mathrm{Dai}^{3^{*}}$ and $\mathrm{Ni} \mathrm{Li}^{3^{*}}$

\begin{abstract}
Background: The effect of sleep duration on cancer risk remains controversial. We aimed to quantify the available evidence on this relationship using categorical and dose-response meta-analyses.

Methods: Population-based cohort studies and case-control studies with at least three categories of sleep duration were identified by searching PubMed, EMBASE, and the Cochrane Library database up to July 2017.

Results: Sixty-five studies from 25 articles were included, involving 1,550,524 participants and 86,201 cancer cases. The categorical meta-analysis revealed that neither short nor long sleep duration was associated with increased cancer risk (short: odds ratio $[O R]=1.01,95 \%$ confidence intervals $[\mathrm{Cl}]=0.97-1.05$; long: $\mathrm{OR}=1.02,95 \% \mathrm{Cl}=0.97-1.07$ ). Subgroup analysis revealed that short sleep duration was associated with cancer risk among Asians ( $\mathrm{OR}=1.36 ; 95 \% \mathrm{Cl}$ : 1.02-1.80) and long sleep duration significantly increased the risk of colorectal cancer ( $\mathrm{OR}=1.21$; $95 \% \mathrm{Cl}$ : 1.08-1.34). The dose-response meta-analysis showed no significant relationship between sleep duration and cancer risk. When treated as two linear piecewise functions with a cut point of $7 \mathrm{~h}$, similar nonsignificant associations were found (per 1-h reduction: $\mathrm{OR}=1.02,95 \% \mathrm{Cl}=0.98-1.07$; per 1-h increment: $\mathrm{OR}=1.003,95 \% \mathrm{Cl}=0.97-1.03$ ).

Conclusion: Categorical meta-analysis indicated that short sleep duration increased cancer risk in Asians and long sleep duration increased the risk of colorectal cancer, but these findings were not consistent in the dose-response meta-analysis. Long-term randomized controlled trials and well-designed prospective studies are needed to establish causality and to elucidate the mechanism underlying the association between sleep duration and cancer risk.
\end{abstract}

Keywords: Cancer incidence, Sleep duration, Categorical meta-analysis, Dose-response meta-analysis

\section{Background}

Cancer is a major public health problem worldwide and a leading cause of death in both more and less economically developed countries; the global burden of cancer is expected to increase because of population growth and aging [1]. Some lifestyle behaviors, such as smoking, alcohol consumption, weight gain, physical inactivity, and delayed or foregone reproduction (e.g., lower parity or later age at first birth) increase cancer risk [2-7].

\footnotetext{
* Correspondence: nli@cicams.ac.cn; daimin2002@hotmail.com

${ }^{\dagger}$ Yuheng Chen and Fengwei Tan contributed equally to this work.

${ }^{3}$ Office for Cancer Early Diagnosis and Treatment, National Cancer Center/

National Clinical Research Center for Cancer/Cancer Hospital, Chinese

Academy of Medical Sciences and Peking Union Medical College, Beijing

100021, China

Full list of author information is available at the end of the article
}

Sleep is an essential component of healthy development and necessary for physical and mental health [8]. Increased attention has been paid to understanding the extent of sleep duration problems at the population level and the associations between these problems and various health outcomes, such as cancer, metabolic syndrome, diabetes mellitus, and all-cause mortality [9-12]. Previous studies indicate that the prevalence of short sleep duration $(<7 \mathrm{~h})$ may have gradually increased over past decades, whereas the prevalence of long sleep duration $(\geq 9 \mathrm{~h})$ has decreased [13]. Epidemiological studies are a valuable way of exploring relationships between sleep and health in the general population [14]. These studies measure sleep and related health variables at a population level, elucidating relationships that may be too subtle to

(c) The Author(s). 2018 Open Access This article is distributed under the terms of the Creative Commons Attribution 4.0 International License (http://creativecommons.org/licenses/by/4.0/), which permits unrestricted use, distribution, and reproduction in any medium, provided you give appropriate credit to the original author(s) and the source, provide a link to the Creative Commons license, and indicate if changes were made. The Creative Commons Public Domain Dedication waiver (http://creativecommons.org/publicdomain/zero/1.0/) applies to the data made available in this article, unless otherwise stated. 
detect in laboratory studies but that are nonetheless useful to society [15]. Epidemiological evidence on the association between sleep duration and cancer risk is controversial, with findings showing inverse [16-18], positive [17, 19-21], and null [22-24] effects. In addition, the dose-response relationship for different quantitative categories of sleep duration in previous studies is unclear [16-18, 24].

The objective of this meta-analysis was to update the evidence on the relationship between sleep duration and cancer risk. We also aimed to explore the quantitative estimates, determine the overall shape of the relationships between sleep duration and cancer incidence, and compare categorical and dose-response meta-analyses.

\section{Methods}

\section{Data sources and searches}

The systematic review and meta-analyses were conducted in accordance with the Preferred Reporting Items for Systematic Reviews and Meta-analyses (PRISMA) Guidelines [25, 26]. We comprehensively identified studies through searching PubMed, EMBASE, and the Cochrane Library database up to July 2017 for both cohort and case-control studies that assessed the association between sleep duration and cancer risk. The following key words were used in the search strategy: (sleep or sleep duration) and (cancer or carcinoma or tumor). The reference lists of retrieved articles were also scanned to locate additional relevant studies.

\section{Study selection criteria}

One investigator $(\mathrm{YHC})$ conducted the initial screening of the article titles and abstracts identified in the first screening process. Two investigators (YHC) and (FWT) independently reviewed the full text of the potentially relevant articles for final inclusion, and any disagreement was resolved through discussion.

For inclusion, studies had to meet the following criteria: (1) original article; (2) cohort study, case-control study, or nested case-control study; (3) estimates and 95\% confidence intervals (CI) (or the raw data to calculate these) for the association between sleep duration and the incident risk of single common cancer or multiple cancers reported in the literature; (4) adult population; and (5) published in English. Studies were excluded if they (1) had duplicated data; (2) were reviews, reports, clinical trials, or genetic and cell studies; or (3) had insufficient data. If more than one article reported data from a single study, the most recent and complete article was included.

\section{Data extraction and quality assessment}

Data were extracted by YHC and independently checked by FWT for accuracy and completeness. Any disagreements were resolved by discussion. For each study, we extracted data on study design; first author's surname; publication year; country of study; study name (cohort studies only); study period; gender of subjects; sample size; types of cancer; number of cases; reference category for sleep; categorization of "short" and "long" sleep duration; fully adjusted relative risks (RRs), odds ratios (ORs), or hazard ratios (HRs) for the associations of both short and long sleep duration with cancer risk; corresponding 95\% confidence intervals (95\% CIs); and the covariates adjusted in the statistical analysis.

Two investigators independently evaluated the quality of the included cohort and case-control studies using the Newcastle-Ottawa Scale (NOS). The selection, comparability, and exposure of each study were broadly assessed and studies were assigned a score from zero to nine. Studies with scores $\geq 7$ were considered of high quality.

\section{Statistical analysis}

We performed categorical and dose-response meta-analyses [27]. Random-effects models were used to pool risk estimates. The adjusted RRs, HRs, ORs, and corresponding 95\% CIs were extracted from the selected studies and used to evaluate the association between sleep duration and the incident risk of any type of cancer. In this meta-analysis, the OR was deemed equivalent to RR and HR, as cancer is a rare outcome [28].

The categorical meta-analysis was conducted by pooling basic classification results of cancer incidence at different levels of sleep duration. In the original articles, sleep duration was assessed using self-report questionnaire measures of habitual sleep duration. We differentiated three levels of sleep duration: short, medium, and long. Short sleep duration was defined as follows in the different articles (in hours per night): $<5 \mathrm{~h}[17,29], \leq 5 \mathrm{~h}$ $[18,20,21,30-32],<6$ h $[22,33-36], \leq 6$ h [16, 23, 37-44], $\leq 6.5 \mathrm{~h}$ [19], and 3-6 h [24]. Long sleep duration was defined as $>7 \mathrm{~h}[35],>8 \mathrm{~h}[36], \geq 8 \mathrm{~h}[19,37], \geq 9 \mathrm{~h}[16,17$, $20-23,29-34,38-44]$, and $\geq 10 \mathrm{~h}[18,24]$ of sleep per night. Medium sleep duration (the reference category) was classified in the studies as $7 \mathrm{~h}[16,20,21,23,31,32$, $37,39,41,44], 8$ h $[18,22,30], 7-8$ h $[17,34,36$, $38,40,42], 7-9$ h [24], 7-7.5 h [19], 6-7 h [35], 6.1$8.9 \mathrm{~h}$ [43], and $7-7.9 \mathrm{~h}$ [29] of sleep per night. For the four studies $[33,35,39,44]$ in which the lowest $(\leq 6 \mathrm{~h}$ or $<6 \mathrm{~h})$ or the highest $(8-9 \mathrm{~h})$ sleep duration level was used as the reference category, we changed the reference group to the medium group $(7 \mathrm{~h}, 6-$ $7 \mathrm{~h}$, or $7-8 \mathrm{~h}$ ), and the RR/OR and the upper and lower CI were calculated for inclusion. Compared with the reference category, the pooled OR and 95\% $\mathrm{CI}$ of cancer risk for both short and long sleep durations were calculated. 
Studies with at least three quantitative categories of short or long sleep duration were also included in doseresponse analyses. Potential nonlinear dose-response relationships between sleep duration and cancer risk were examined using a restricted cubic splines model with three knots at the 10th, 50th, and 90th percentiles of the distribution. We assigned the median or mean sleep duration in each category to the corresponding OR for each study. If the mean or median duration per category was not reported, the midpoint of the upper and lower boundaries in each category was assigned. For the open-ended risk factor classes (e.g., $<5$ or $>10$ ), we assigned a value following the algorithms suggested by Il'yasova et al. [45], choosing those algorithms that yielded the most plausible results for sleep duration. For upper open-ended categories, we assigned the value of the lower bound plus the width of the previous (second-to-highest) interval. For lower open-ended categories, we assigned the value of the upper bound minus half the width of the next (second-to-lowest) interval [46]. $P_{\text {nonlinearity }}$ was identified by testing the null hypothesis that the estimated value of the second spline was equal to zero. If the null hypothesis did not hold, we conducted a linear dose-response meta-analysis to test the cancer risk associated with each additional hour of sleep. Otherwise, a nonlinear dose-response meta-analysis was conducted to identify the cancer risk associated with each hour. For linearity, if a U-, J-, or S-shape curve or a significant nonlinear shape association was observed, we treated the slope as two piecewise linear functions with the cut point of $7 \mathrm{~h}$ to show the separate linear trends [47]. All pooled outcome measures were determined using random-effects models, as described by DerSimonian and Laird, as these models produce more conservative results than fixed-effects models.

Heterogeneity among studies was estimated using Cochran's Q test (reported with a $x^{2}$-value and $P$-value) and the $I^{2}$ statistic $[48,49]$. For the Q test, a $P$-value of less than 0.1 was considered to indicate the presence of heterogeneity. The $I^{2}$ statistic was used to test whether the proportion of total variation in the estimates could be explained by heterogeneity rather than chance. $I^{2}$ values of 25,50 , and $75 \%$ were considered evidence of low, moderate, and high heterogeneity, respectively.

Subgroup analyses were carried out by study region (America, Asia, and Europe), gender (women and men), the definition of short or long sleep duration (" $\leq 6$ or $\leq 5$ " and " $\geq 9$ or $\geq 10$ "), the definition of the reference category ( $7 \mathrm{~h}-8 \mathrm{~h}$ or $7 \mathrm{~h}$ ), the definition of short or long sleep duration versus the reference category (" $\leq 6$ vs.7" and " $\geq 9$ vs. 7 "), study quality score $(\geq 7$ and $<7)$ and cancer type to minimize heterogeneity among the included studies. In addition, we estimated the risk of sex hormone-related cancer (including breast cancer, endometrial cancer, ovarian cancer, and prostate cancer, which are associated with sex hormone regulation) $[50,51]$. In each specific population, the effects of short or long sleep duration on cancer risk were evaluated. We also conducted a sensitivity analysis by sequentially removing each individual study from the meta-analysis.

We visually inspected the symmetry of the funnel plots and performed the Begg regression test and Egger's linear regression test to assess the possibility of publication bias $[52,53]$. For funnel plot asymmetry, a contour-enhanced funnel plot of the effect size was examined to test for publication bias [54]. All statistical analyses were performed using Stata software (version 11.0; StataCorp, College Station, TX, USA). A $P$-value $<0.05$ was considered statistically significant.

\section{Results}

\section{Study selection}

The literature searches identified a total of 5288 articles: 1321 from PubMed, 3418 from EMBASE, and 549 from the Cochrane Library (Fig. 1). After the initial screening of titles and the exclusion of duplicates, 95 articles were retrieved for further evaluation. The full text review showed that 1 article used a duplicated study population [55], 2 investigated the association between sleep duration and colorectal adenoma [56, 57], 13 were not original articles, and 54 were unrelated to the exposure or outcomes of interest. After excluding these articles, 25 articles that met the inclusion and exclusion criteria were used in this meta-analysis, which we performed in accordance with the guidelines of the PRISMA Statement [58] (Additional file 1).

\section{Study characteristics}

A summary of the study characteristics is shown in Additional file 2. We identified 21 cohort articles involving 79,885 cases and 1,488,349 participants, and 4 case-control studies involving 6316 cases and 55,859 control subjects. Two of the twenty-one cohort articles reported results for three and six different types of cancer [16, 24], two articles reported the results for men and women [21, 23], and one article reported results for 15 different cancer types among men and 17 among women [17]. These were considered as separate studies in the analysis. Therefore, a total of 25 articles including 65 studies were included in the final meta-analysis. Descriptive data for the 65 included studies are summarized in Additional file 2. Twenty-two studies $[17-19,21,23,30,37,40]$ were conducted with men, forty-two [16, 17, 20-24, 29, 31-33, 35, 36, 38, 39, 41-44] with women, and one [34] with both men and women. Fifty-five studies were conducted in the United States [16-18, 20-24, 29, 31-33, 37-39], three in Japan [35, 40, 41], two in Finland [19, 42], one in Australia [36], 


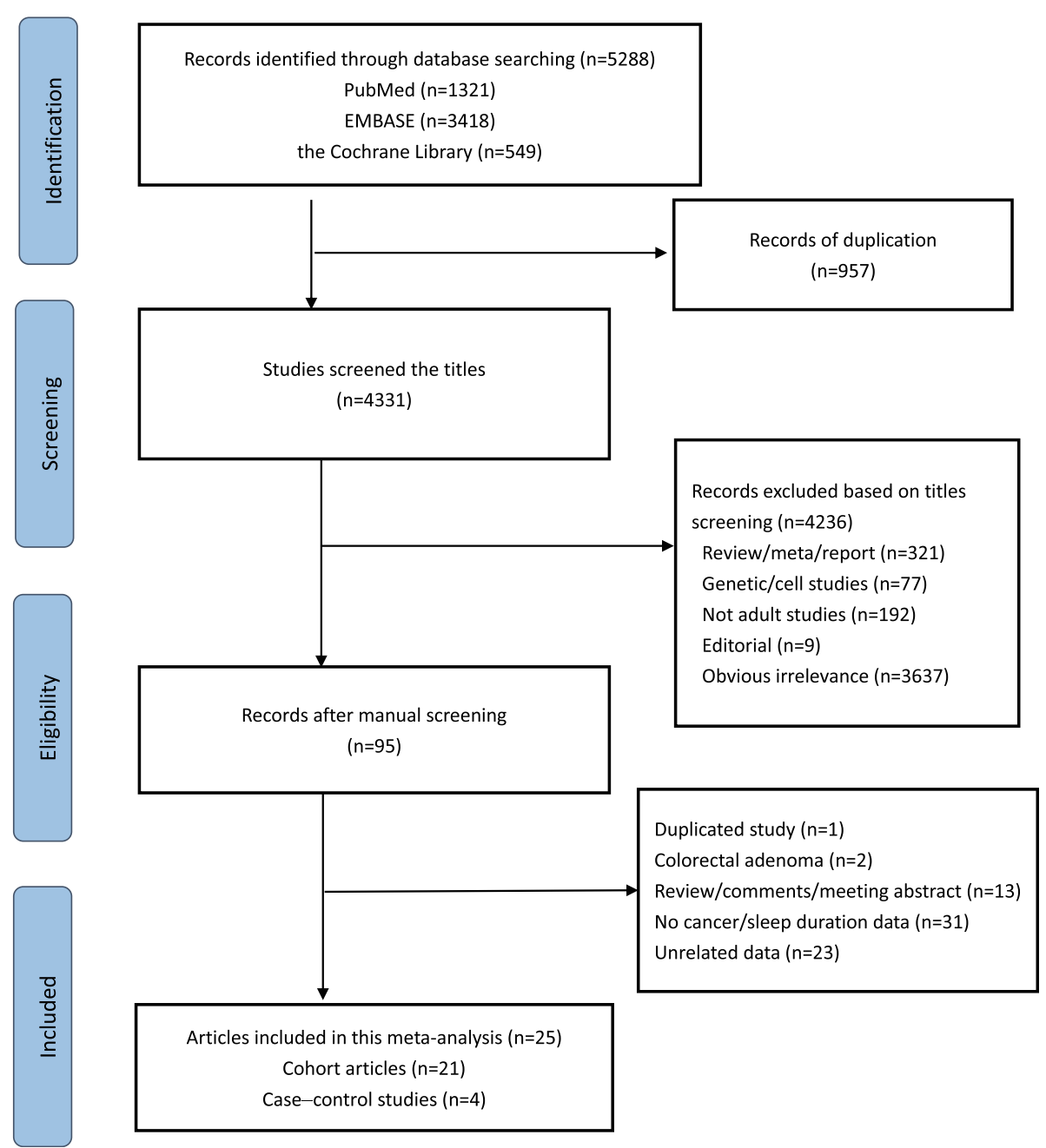

Fig. 1 Flow chart showing the number of articles identified at each stage of the search

one in China [43], one in Europe [34], one in Singapore [44], and one in Sweden [30]. Study size ranged from 1975 to 173,327 participants. Duration of sleep was assessed using either questionnaires or interviews that measured self-reported habitual sleep duration. All studies ascertained cancer by physician diagnosis, medical records, or cancer registry-based sources. In terms of cancer subtypes, the association between sleep duration and breast cancer, skin cancer, colorectal cancer, lung cancer, prostate cancer, endometrial cancer, ovarian cancer, thyroid cancer, other cancers and undefined cancers were reported by 12 $[17,22,24,29,31-33,36,41-44]$, six [16, 23, 24], six $[17,20,21,24]$, five $[17,19,24,37]$, four $[17,18,40]$, $3[17,24,39]$, three $[17,24,35]$, three [17, 38], 22 [17], and one [34] study/studies, respectively. Using the Newcastle-Ottawa Scale, the methodological quality of the studies was judged as high, with a mean score of $7.44 \pm 0.65$, a median of 8 , a range of 6 to 8 points, and most of the studies scoring $\geq 7$ (Additional files 3-4).

\section{Categorical meta-analysis \\ Short sleep duration and cancer risk}

The combined OR comparing the shortest categories with the reference category of sleep duration was 1.00 (95\% CI: 0.96-1.04) for the 61 cohort studies, with low to moderate heterogeneity $\left(P=0.02, I^{2}=28.1 \%\right)$, and 1.14 (95\% CI: 0.94-1.37) for the 4 case-control studies, with low to moderate heterogeneity $\left(P=0.22, I^{2}=32.3 \%\right)$ (Fig. 2). Combining the two types of study designs resulted in an overall combined OR of 1.01 (95\% CI: 0.971.05, $P$ for heterogeneity: $\left.P=0.015, I^{2}=29.8 \%\right)$. Exclusion of a single study did not substantially influence the combined estimates for the cohort or case-control studies (Additional file 5). No publication bias was detected for short sleep duration and cancer risk (Egger's test: $P=0.051$; Begg's test: $P=0.275)$ in the selected 


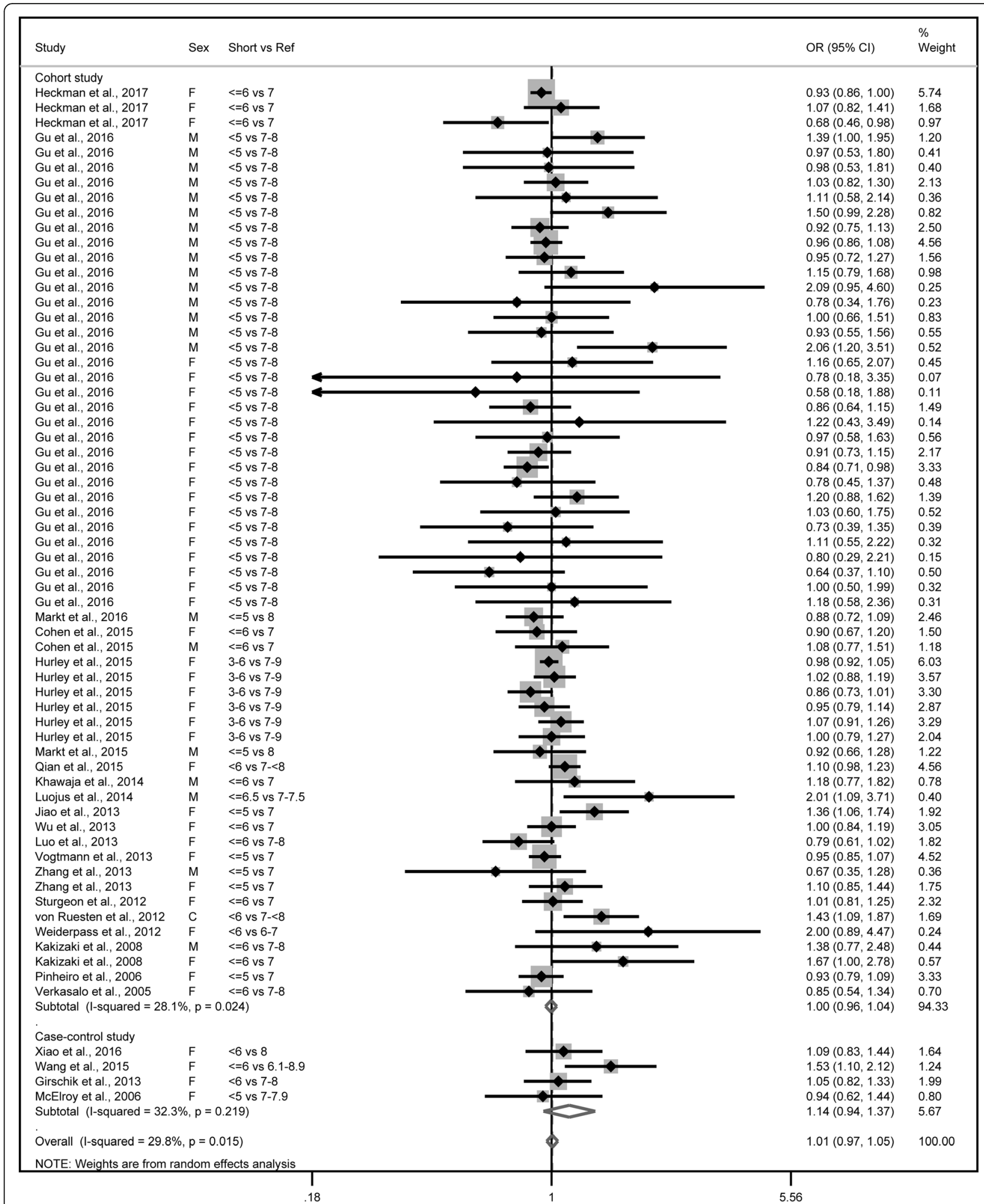

Fig. 2 Forest plot of association between short sleep duration and cancer risk. Box sizes reflect the weights of studies included in the meta-analysis, horizontal lines are the $95 \% \mathrm{Cls}$, and the summary OR is represented by the diamond. OR: odds ratio, Cl: confidence interval 
studies (Fig. 3). As shown in Table 1, short sleep duration was associated with cancer risk only in the Asian population $(\mathrm{OR}=1.36$; 95\% CI: 1.02-1.80). Cancer risk did not vary substantially by sleep duration in most subgroups.

\section{Long sleep duration and cancer risk}

The combined OR comparing the longest sleep categories and the reference category of sleep duration was 1.00 (95\% CI: $0.95-1.06)$ for the 61 cohort studies with low to moderate heterogeneity $\left(P=0.02, I^{2}=28.3 \%\right)$ and 1.15 (95\% CI: 0.96-1.38) with moderate heterogeneity $\left(P=0.10, I^{2}=51.2 \%\right)$ for the 4 case-control studies (Fig. 4). Combining the two types of study designs resulted in an overall combined OR of 1.02 (95\% CI: 0.97-1.07, $P$ for heterogeneity: $\left.P=0.01, I^{2}=31.3 \%\right)$. Exclusion of a single study did not substantially influence the combined estimates for the cohort or case-control studies (Additional file 6). No publication bias was detected for long sleep duration and cancer risk (Egger's test: $P=0.935$; Begg's test: $P=0.305)$ in the selected studies (Fig. 5). As shown in Table 1, in terms of cancer type, a significant association was found between long sleep duration and colorectal cancer $(\mathrm{OR}=1.21$; 95\% CI: $1.08-$ 1.34). No significant associations were observed when the data were stratified by study region, gender, or definition of sleep duration.

\section{Dose-response meta-analysis}

Sixty-one population-based cohort studies and four case-control studies, including 86,201 cases among $1,550,524$ participants, were combined in the dose-response meta-analysis of sleep duration and cancer risk. As shown in Fig. 6, the nonlinear $(P=0.24)$ dose-response analysis indicated no relationship between sleep

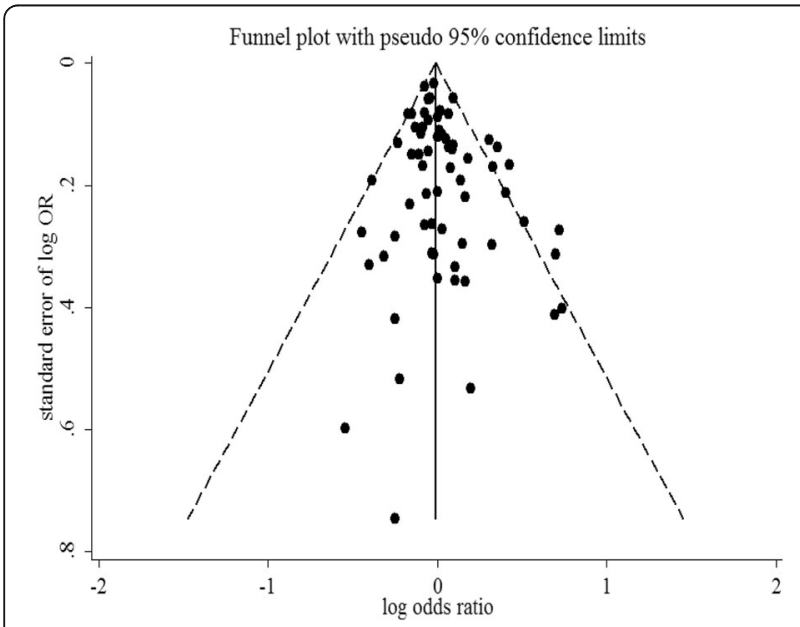

Fig. 3 Funnel plot of studies evaluating the association between short sleep duration and cancer risk. Dotted lines indicate 95\% pseudo-confidence interval. SE: standard error; OR: odds ratio duration and cancer risk (Additional file 7). However, a linear relationship $(P=0.84)$ was found, which suggested that increasing sleep duration (in 1-h increments) was not associated with cancer risk $(\mathrm{OR}=0.999,95 \% \mathrm{CI}=$ 0.993-1.006). When treated as two piecewise linear functions, among people who slept less than $7 \mathrm{~h}$ per night, a 1-h reduction in sleep duration was not associated with an increase in cancer risk $(\mathrm{OR}=1.021,95 \% \mathrm{CI}$ $=0.979-1.066$ ), and the results for people with sleep durations of more than $7 \mathrm{~h}$ were similar $(\mathrm{OR}=1.003$, 95\% CI $=0.972-1.034)$.

Using the available data, we examined potential effect modifications by study region, gender, study design (cohort study and case-control study), study quality score, and cancer type. The association was not appreciably modified by study region, gender, study quality, or study design. When stratified by cancer type, we observed a J-shaped curvilinear association, with the lowest colorectal cancer risk at a sleep duration of about $7 \mathrm{~h}$ per night $(P=0.005$ for nonlinearity, Additional file 8$)$. When treated as two piecewise linear functions, for people who slept for less than $7 \mathrm{~h}$ per night, a 1-h sleep duration reduction was not associated with colorectal cancer risk $(\mathrm{OR}=0.994,95 \% \mathrm{CI}=0.889-1.113)$; results for those with sleep durations longer than $7 \mathrm{~h}$ were similar $(\mathrm{OR}=$ $1.064,95 \% \mathrm{CI}=0.978-1.157)$.

\section{Discussion}

This study provided a comprehensive systematic review of the literature and quantitative estimates of the associations of short and long sleep duration with the risk of cancer in population-based observational studies. The findings showed that neither short nor long sleep duration was significantly associated with cancer risk, regardless of the examined categories in the meta-analysis or dose-response meta-analysis. Interestingly, the subgroup analysis revealed that short sleep duration was associated with increased risk of cancer in Asian populations (this finding was based on four cohort and one case-control study including 1779 incident cases) and long sleep duration was associated with increased risk of colorectal cancer (this finding was based on six cohort studies including 8099 incident cases).

\section{Comparison with previous reviews and meta-analyses}

Most previous reviews and meta-analyses of studies on sleep duration and cancer risk have reported null findings or shown that longer sleep duration might be a risk factor for colorectal cancer, which is consistent with our findings [59-61]. A meta-analysis published in 2013 found that neither extremely short sleep duration nor long sleep duration was statistically associated with an increased risk of cancer in a categorical meta-analysis (short: $\mathrm{HR}=1.06,95 \% \mathrm{CI}=0.92-1.23$; long: $\mathrm{HR}=0.91$, 
Table 1 Subgroup analyses of association between sleep duration and cancer risk

\begin{tabular}{|c|c|c|c|c|c|c|c|c|}
\hline \multirow[t]{2}{*}{ Subgroups } & \multicolumn{4}{|c|}{ Short sleep duration } & \multicolumn{4}{|c|}{ Long sleep duration } \\
\hline & $\bar{n}$ & OR $(95 \% \mathrm{Cl})$ & $1^{2}(\%)$ & $\overline{P_{\text {Heterogeneity }}}$ & $\bar{n}$ & OR $(95 \% \mathrm{Cl})$ & $1^{2}(\%)$ & $\overline{P_{\text {Heterogeneity }}}$ \\
\hline \multicolumn{9}{|l|}{ Regions } \\
\hline USA & 55 & $0.98(0.95-1.02)$ & 13.2 & 0.207 & 55 & $1.01(0.97-1.06)$ & 7.4 & 0.320 \\
\hline Asia & 5 & $1.36(1.02-1.80)$ & 58.1 & 0.049 & 5 & $0.75(0.44-1.27)$ & 82.0 & $<0.001$ \\
\hline Europe & 4 & $1.19(0.84-1.67)$ & 66.7 & 0.029 & 4 & $0.95(0.64-1.41)$ & 70.6 & 0.017 \\
\hline \multicolumn{9}{|l|}{ Gender } \\
\hline Women & 42 & $0.98(0.94-1.03)$ & 22.4 & 0.102 & 42 & $1.02(0.96-1.09)$ & 30.0 & 0.037 \\
\hline Men & 22 & $1.06(0.96-1.16)$ & 29.8 & 0.094 & 22 & $1.02(0.94-1.11)$ & 33.3 & 0.066 \\
\hline \multicolumn{9}{|c|}{ Definition of short or long sleep duration } \\
\hline$\leq 6$ or $\geq 9$ & 14 & $1.01(0.92-1.11)$ & 44.4 & 0.037 & 54 & $1.01(0.95-1.06)$ & 27.7 & 0.034 \\
\hline$\leq 5$ or $\geq 10$ & 6 & $1.00(0.87-1.15)$ & 51.1 & 0.069 & 7 & $1.14(0.87-1.49)$ & 48.7 & 0.069 \\
\hline \multicolumn{9}{|l|}{ Definition of reference category } \\
\hline $7-8 \mathrm{~h}$ or $7 \mathrm{~h}$ & 51 & $1.00(0.95-1.05)$ & 24.6 & 0.061 & 51 & $1.00(0.95-1.05)$ & 21.9 & 0.088 \\
\hline \multicolumn{9}{|c|}{ Definition of short sleep or long duration versus reference category } \\
\hline$\leq 6$ vs. 7 or $\geq 9$ vs. 7 & 9 & $0.98(0.90-1.07)$ & 27.0 & 0.204 & 15 & $0.99(0.90-1.08)$ & 43.3 & 0.038 \\
\hline \multicolumn{9}{|l|}{ Study quality score } \\
\hline$\geq 7$ & 58 & $1.02(0.97-1.07)$ & 33.9 & 0.007 & 58 & $1.01(0.95-1.06)$ & 32.4 & 0.011 \\
\hline$<7$ & 7 & $0.97(0.93-1.02)$ & 0.00 & 0.627 & 7 & $1.14(0.95-1.37)$ & 26.5 & 0.226 \\
\hline \multicolumn{9}{|l|}{ Cancer type } \\
\hline Sex hormone-related cancers & 22 & $0.99(0.94-1.04)$ & 32.9 & 0.069 & 22 & $0.97(0.89-1.06)$ & 51.0 & 0.003 \\
\hline Breast cancer & 12 & $1.00(0.94-1.08)$ & 46.1 & 0.040 & 12 & $1.02(0.92-1.12)$ & 51.0 & 0.021 \\
\hline Skin cancer & 6 & $0.93(0.88-1.00)$ & 0.0 & 0.481 & 6 & $0.92(0.78-1.10)$ & 19.4 & 0.287 \\
\hline Colorectal cancer & 6 & $1.05(0.92-1.19)$ & 38.3 & 0.151 & 6 & $1.21(1.08-1.34)$ & 0.0 & 0.555 \\
\hline Endometrial cancer & 3 & $0.98(0.82-1.17)$ & 44.3 & 0.135 & 3 & $1.06(0.83-1.34)$ & 0.0 & 0.589 \\
\hline Lung cancer & 5 & $1.04(0.88-1.22)$ & 46.1 & 0.115 & 5 & $1.01(0.83-1.23)$ & 41.6 & 0.144 \\
\hline Ovarian cancer & 3 & $1.05(0.72-1.53)$ & 44.3 & 0.166 & 3 & $0.84(0.46-1.52)$ & 60.5 & 0.079 \\
\hline Prostate cancer & 4 & $0.95(0.86-1.04)$ & 0.0 & 0.540 & 4 & $0.75(0.54-1.05)$ & 70.9 & 0.016 \\
\hline Thyroid cancer & 3 & $1.11(0.64-1.93)$ & 65.0 & 0.058 & 3 & $0.95(0.63-1.45)$ & 0.0 & 0.539 \\
\hline
\end{tabular}

Asia: China, Japan and Singapore; Europe: Europe, Finland and Sweden

Sex hormone-related cancers: included breast cancer, endometrial cancer, ovarian cancer, and prostate cancer

Skin cancer: included basal cell skin cancer, squamous cell skin cancer, and melanoma

$95 \% \mathrm{CI}=0.78-1.07)$. The same meta-analysis, which included 13 cohort studies, found no significant dose-response relationship $(P=0.51)$ between sleep duration and cancer [61]. A meta-analysis of 10 cohort studies by $\mathrm{Lu}$ et al. [60] categorized sleep duration into three groups: short, moderate, and long. They concluded that neither short nor long sleep duration was statistically associated with increased risk of cancer (short: $R R=1.05$, 95\% CI $=0.90-1.24$; long: $\mathrm{RR}=0.92,95 \% \mathrm{CI}=0.76-1.12$ ) and that long sleep duration was positively associated with colorectal cancer $(\mathrm{RR}=1.29,95 \% \mathrm{CI}=1.09-1.52)$. Erren et al. [59] reported that the combined adjusted RR was $1.08(95 \% \mathrm{CI}=1.03-1.13)$ for colorectal cancer and $1.11(95 \% \mathrm{CI}=1.00-1.22)$ for lung cancer, but this analysis did not examine the dose-response relationships. We conducted a flexible, categorical, and dose-response meta-analysis and treated the slope as two piecewise linear functions with the cut point of $7 \mathrm{~h}$ to show the linear trends. Therefore, the present study might provide the most comprehensive assessment and robust evidence to date on the relationship between sleep duration and cancer risk.

\section{Potential mechanisms}

The etiology of cancer is multifactorial; genetic, metabolic, environmental, behavioral, and social or cultural factors are major contributors. Although the exact mechanism remains unknown, there are several possible pathways that could explain the association between sleep duration and cancer risk. First, the melatonin hypothesis proposes that shorter sleep duration is associated with decreased levels of melatonin [62], and 


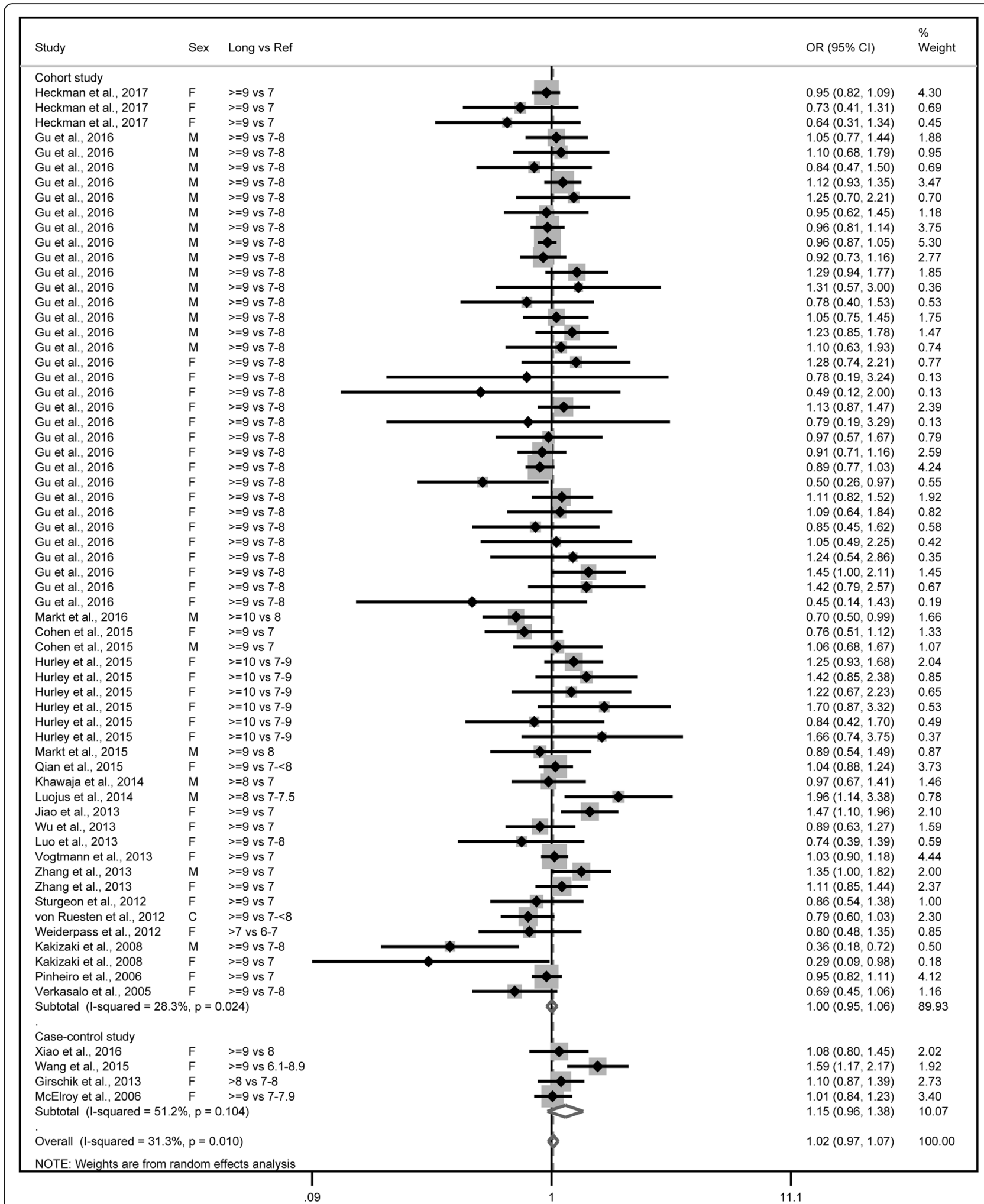

Fig. 4 Forest plot of association between long sleep duration and cancer risk. Box sizes reflect the weights of studies included in the meta-analysis, horizontal lines are the $95 \% \mathrm{Cls}$, and the summary OR is represented by the diamond. OR: odds ratio, Cl: confidence interval 


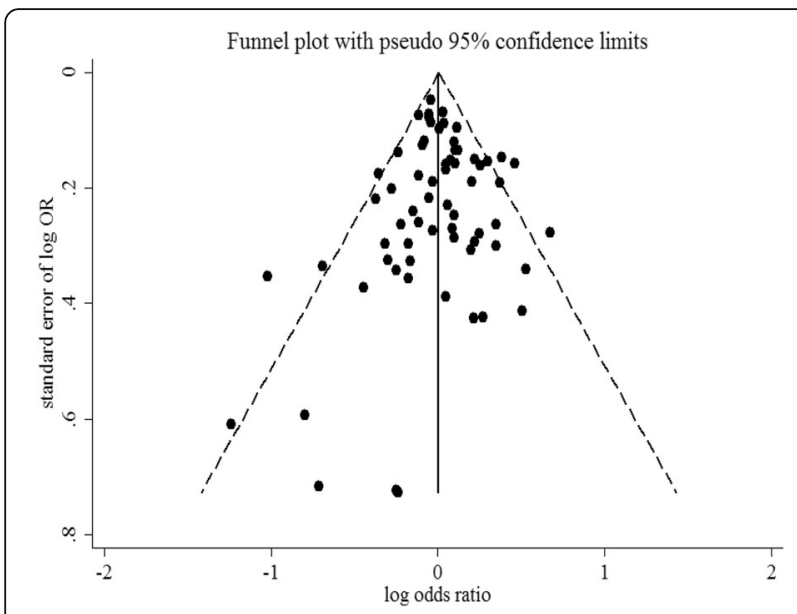

Fig. 5 Funnel plot of studies evaluating the association between long sleep duration and cancer risk. Dotted lines indicate 95\% pseudo-confidence interval. SE: standard error; OR: odds ratio

melatonin has been found to suppress the initiation phase of tumorigenesis and inhibit the proliferation of human cancer cell lines in experimental studies [14,63]. Numerous studies have demonstrated the association of sex hormones with the development and progression of various types of cancer, including cancers of the breast, endometrium, ovary, and prostate [64-70]. Moreover, melatonin may modulate sex hormone production by interacting with estrogen-signaling pathways through different mechanisms [71, 72]. A recent dose-response meta-analysis by Yang et al. has found that an increase in urinary aMT6s of $15 \mathrm{ng} / \mathrm{mg}$ creatinine is associated with a $14 \%$ reduced risk of breast cancer, especially in post-menopausal women [73]. Therefore, melatonin might play a role in the progression of sex hormone-related cancers. Although several studies have evaluated the

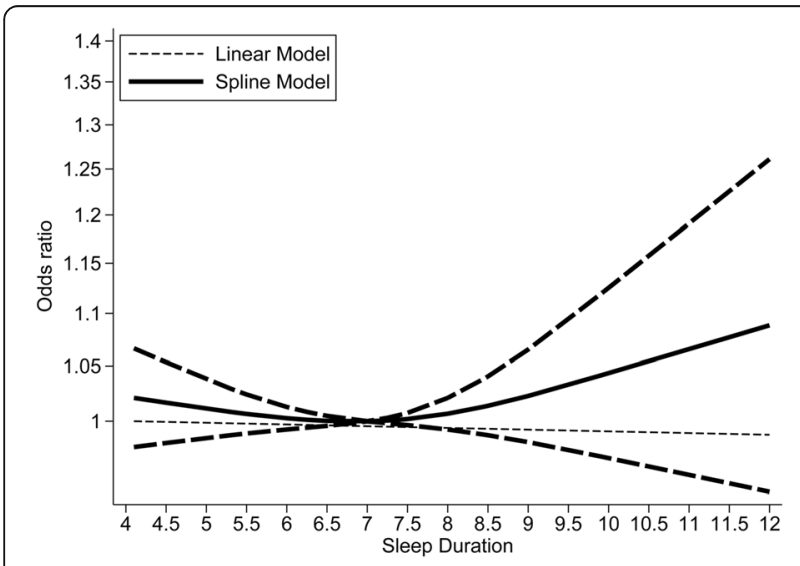

Fig. 6 Nonlinear dose-response analyses of sleep duration and cancer risk. The solid line and the long-dashed line represent the estimate odds ratios and their $95 \%$ confidence intervals. Seven hours of sleep per night was used as the reference interrelation between melatonin and sex hormone levels, the interpretations of their findings are inconsistent [7476]. The second possible mechanism is impaired immune function [77]. Both laboratory studies of acute sleep deprivation and observational studies of poor sleepers have reported that sleep duration changes may lead to a suppression of immune function and a shift in the balance of cytokine production $[78,79]$. The third possible mechanism is disruption of circadian rhythms. Disruption of circadian physiology owing to an annual decrease in sleep duration or sleep disturbances may result in impaired glucose, reduced appetite control [14], and various gastrointestinal diseases, such as irritable bowel syndrome, gastroesophageal reflux disease, or peptic ulcer disease. In addition, circadian disruption can promote tumorigenesis in the liver and gastrointestinal tract [80]. The fourth mechanism involves metabolic pathways related to obesity, which is a risk factor for several cancers [81]. The decrease of sleep duration may be a stress response to chronic stress and unhealthy emotions. Chronic stress plays a significant role in cancer incidence, and depression is a risk factor for cancer onset and cancer progression [82, 83].

Our findings indicate that long sleep duration is an additional behavioral risk factor for colorectal cancer. The proposed mechanism is that sleep may influence cancer risk via alterations in levels of appetite-regulating hormones, such as leptin and ghrelin [84, 85], leading to increased appetite and subsequent obesity [84, 86-88]. The association between sleep duration and colorectal cancer may also be explained by comorbidities [89] and residual confounding. For instance, other mental or physiological disorders, low socioeconomic status, low levels of physical activity, and undiagnosed chronic comorbid conditions may be correlated with long sleep duration; these factors could confound the association between sleep duration and cancer incidence [61, 90].

We also performed a subgroup analysis by study population. Short sleep duration was strongly associated with increased cancer incidence in Asian participants but not in American and European participants. Several possible pathways could explain the relationship between short sleep duration and increased cancer incidence in Asians. First, there may be differences in melatonin secretion patterns between Asians and Americans [91]. Wetterberg et al. have published two studies comparing urinary melatonin in women in Asian (Japanese) and Caucasian (American) populations. Both studies found significantly lower levels of melatonin excretion in Japanese women compared with American women [92, 93]. Although it was not possible to investigate these effects in the present study, ethnic background should be considered a variable of interest in future studies. Second, differences in sleep patterns (e.g., daytime naps, the use of sleep 
medication, and sleeping alone or with a partner) across different countries might have affected the results [94]. In a Chinese population-based study of 452,829 adults aged 30-79 years, $20.3 \%$ of participants had daytime naps all year round and $40.1 \%$ had daytime naps in summer [95]. Napping increases sleep duration and may be correlated with sleep disturbances or poor sleep [59]. In addition, the results of our meta-analysis should be viewed with caution owing to heterogeneity caused by discrepancies in sample sizes, sample characteristics, response rates, and sleep duration measures. Additional studies are needed for reliable quantification of this association and to evaluate whether these factors contribute to region-related differences.

\section{Strengths and limitations}

Our study addressed the limitations of previous research and had several strengths. First, this meta-analysis was based on up-to-date literature and presented the largest synthesis to date of prospective cohort studies and case-control studies with large sample sizes, which increased the statistical power to detect potential associations. Second, data for the pooled analysis were derived from fully adjusted models in the primary studies, which should reduce the likelihood of confounding. Moreover, the combined use of categorical and dose-response meta-analyses provided more information. Linear and nonlinear relationships were also tested to assess the dose-response relationship, and we performed subgroup and sensitivity analyses on potential confounders. The methodological features of our study enhance the quality of our results and strengthen the validity of the conclusions.

Several limitations of this meta-analysis should be considered. First, meta-analyses are greatly influenced by the quality of the individual studies included. Nearly all the included studies relied on self-reports of sleep duration collected via questionnaires or interviews; this type of data may not fully or accurately capture actual sleep duration. Additionally, sleep duration was classified differently across the original studies, and the differences in the reference groups were particularly large. Second, this meta-analysis summarizes results from both prospective cohort and case-control studies. However, there are many differences between these two types of study design, such as different statistical estimates (HRs, RRs, or ORs) and different biases. The previous two limitations may have led to heterogeneity in our meta-analysis. Thus, subgroup analyses, sensitivity analyses, and random-effects models were generated to examine sources of heterogeneity. Third, most of the studies assessed sleep duration at a single point in time; this method might not accurately reflect the sustained effects of sleep duration over time when relating them to the long-term development of cancer. Fourth, the available data were limited for several studies, which might have led to a loss of statistical power for some of the subgroup results. In the subgroup analysis of cancer types and study population, the categorical and dose-response analyses produced inconsistent results. It is possible that there was insufficient statistical power to detect a dose-response effect in the different groups; studies with larger subgroup sample sizes are needed to validate these associations. Fifth, most studies reported data for only breast, skin, colorectal, lung, prostate, endometrial, ovarian, and thyroid cancers, except one European study that reported data for all cancer types [34]. Therefore, the present findings cannot be generalized to all cancer types.

\section{Implications for practice and research}

Because of limited evidence and a lack of consensus on the effects of sleep duration on cancer risk, we believe that the following aspects warrant close attention in future investigations of the association between sleep duration and cancer risk $[12,59,96]$ :

(1) Study design. Additional targeted biological research is needed to determine the exact mechanisms underlying the association between sleep duration and cancer risk, and population-based long-term epidemiological studies are needed to explore the link between specific (precisely measured) sleep durations and cancer risk.

(2) Measurement and assessment of sleep duration. Further epidemiological studies should be conducted using electronic wearable devices to obtain precise, reliable, and scalable objective measures of sleep duration (how long), sleep timing (when), sleep quality (good or bad), and location (longitude and latitude). Such studies should also assess, as directly as possible, the effects of sleep duration and changes in sleep duration on cancer incidence and development during long-term follow-up.

(3) Confounding bias. The presence of confounding variables is a limitation of many studies included in this meta-analysis; therefore, future studies should control for the following:

- The need to sleep with a light on (always to never);

- Sleep environmental factors (presence of young children, population density, place where the subjects sleeps, level of noise at the sleeping place);

- Sleep quality assessment (insomnia, snoring, sleep apnea, sleep deprivation);

- Sleep culture (watching TV/drinking in bed, daytime napping); and

- The use of melatonin supplementation 
(4) Chronobiological variables. Further experimental studies that focus on melatonin, hormonal rhythms, and clock gene expressions are needed to interpret the complex relationships between sleep and cancer.

\section{Conclusions}

Our findings indicate that neither short nor long sleep duration was significantly associated with cancer risk, although short sleep duration slightly increased cancer risk among Asians and long sleep duration slightly increased the risk of colorectal cancer. Large-scale, well-designed prospective studies are required to further investigate the observed association. Long-term randomized controlled trials are needed to establish causality and to elucidate the mechanisms underlying the association between sleep duration and cancer risk.

\section{Additional files}

Additional file 1: PRISMA 2009 Checklist. (DOCX $25 \mathrm{~kb}$ )

Additional file 2: Characteristics of studies included in the meta-analysis. (DOCX $39 \mathrm{~kb}$ )

Additional file 3: Study quality of cohort studies included in the analysis of sleep duration and cancer risk. (DOCX $21 \mathrm{~kb}$ )

Additional file 4: Study quality of case-control studies included in the analysis of sleep duration and cancer risk. (DOCX $20 \mathrm{~kb}$ )

Additional file 5: Sensitivity analysis of sleep duration and cancer risk, shortest vs. reference analysis. (PDF $194 \mathrm{~kb}$ )

Additional file 6: Sensitivity analysis of sleep duration and cancer risk, longest vs. reference analysis. (PDF $186 \mathrm{~kb}$ )

Additional file 7: Association between sleep duration and cancer risk from nonlinear dose-response analysis. (DOCX $20 \mathrm{~kb}$ )

Additional file 8: Nonlinear dose-response analyses of sleep duration and colorectal cancer risk. (PDF $79 \mathrm{~kb}$ )

\section{Abbreviations}

95\% Cl: 95\% confidence interval; HR: Hazard ratio; NOS: Newcastle-Ottawa Scale (NOS); OR: Odds ratio; PRISMA: Preferred reporting items for systematic reviews and meta-analyses; RR: Relative risk

\section{Acknowledgements}

We thank the useful discussion by our research team and Prof. Ni Li, for the study design and critical review. We would also like to thank the reviewers whose comments and suggestions improved this manuscript.

\section{Funding}

This work was funded by National Key R\&D Program of China (Grant no: 2016YFC0905400, 2016YFC1302500, 2017YFC0907900, 2018YFC1315000), National Natural Science Foundation of China (Grant no: 81871885 , 81673265), Training Programme Foundation for the Talents in Beijing City (Grant no: 2017000021223TD05), Beijing Municipal Science and Technology Project (Grant no: D171100002617001), Special Foundation for Central Committee Health Care (Grant no: W2017BJ39), and PUMC Youth Fund (Grant no: 2017320013).

\section{Availability of data and materials}

All data generated or analyzed during this study are included in this published article and its Additional files.

\section{Authors' contributions}

YHC designed the study. NL and MD directed YHC and FWT work on the meta-analysis. YHC and FWT conducted the analyses and YHC wrote the first full draft of the manuscript. FWT updated the analysis. All authors contributed to the conceptualization of the paper, critically reviewed all drafts and contributed to the revision of the article. All authors read and approved the final manuscript.

\section{Ethics approval and consent to participate}

Not applicable.

\section{Consent for publication}

Not applicable.

\section{Competing interests}

The authors declare that they have no competing interests.

\section{Publisher's Note}

Springer Nature remains neutral with regard to jurisdictional claims in published maps and institutional affiliations.

\section{Author details}

${ }^{1}$ Cancer Foundation of China, National Cancer Center/National Clinical Research Center for Cancer/Cancer Hospital, Chinese Academy of Medical Sciences and Peking Union Medical College, Beijing 100021, China.

${ }^{2}$ Department of Thoracic Surgery, National Cancer Center/National Clinical Research Center for Cancer/Cancer Hospital, Chinese Academy of Medical Sciences and Peking Union Medical College, Beijing 100021, China. ${ }^{3}$ Office for Cancer Early Diagnosis and Treatment, National Cancer Center/National Clinical Research Center for Cancer/Cancer Hospital, Chinese Academy of Medical Sciences and Peking Union Medical College, Beijing 100021, China. ${ }^{4}$ Henan Office for Cancer Control and Research, the Affiliated Cancer Hospital of Zhengzhou University, Henan Cancer Hospital, Zhengzhou 450008, China.

Received: 11 May 2018 Accepted: 31 October 2018

Published online: 21 November 2018

\section{References}

1. Torre LA, Bray F, Siegel RL, Ferlay J, Lortet-Tieulent J, Jemal A. Global cancer statistics, 2012. CA Cancer J Clin. 2015;65(2):87-108.

2. Jung $\mathrm{K}$, Jeon C, Jee SH. Smoking effect on lung cancer: ethnic difference and smoking paradox. Epidemiol Health. 2016;38:e2016060.

3. Wang X, Cheng W, Li J, Zhu J. A meta-analysis of alcohol consumption and thyroid cancer risk. Oncotarget. 2016;7(34):55912-23.

4. Wolin KY, Colditz GA. Can weight loss prevent cancer? Br J Cancer. 2008;99(7):995-9.

5. Sanchis-Gomar F, Lucia A, Yvert T, Ruiz-Casado A, Pareja-Galeano H, SantosLozano A, et al. Physical inactivity and low fitness deserve more attention to Alter cancer risk and prognosis. Cancer Prev Res. 2015;8(2):105-10.

6. Wu AH, Pearce CL, Lee AW, Tseng C, Jotwani A, Patel P, et al. Timing of births and oral contraceptive use influences ovarian cancer risk. Int J Cancer. 2017;141(12):2392-9.

7. Milne RL, Osorio A, Ramón Y, Cajal T, Baiget M, Lasa A, Diaz-Rubio E, et al. Parity and the risk of breast and ovarian cancer in BRCA1 and BRCA2 mutation carriers. Breast Cancer Res Treat. 2010;119(1):221-32.

8. Chaput J, Gray CE, Poitras VJ, Carson V, Gruber R, Olds T, et al. Systematic review of the relationships between sleep duration and health indicators in school-aged children and youth. Appl Physiol Nutr Metab. 2016;41(6(Suppl. 3)):S266-82.

9. St-Onge M, Grandner MA, Brown D, Conroy MB, Jean-Louis G, Coons M, et al. Sleep duration and quality: impact on lifestyle behaviors and cardiometabolic health: a scientific statement from the American Heart Association. Circulation. 2016;134(18):e367-86.

10. Shan Z, Ma H, Xie M, Yan P, Guo Y, Bao W, et al. Sleep duration and risk of type 2 diabetes: a meta-analysis of prospective studies. Diabetes Care. 2015;38(3):529-37

11. Qin $Y$, Zhou $Y$, Zhang $X$, Wei $X$, He J. Sleep duration and breast cancer risk: a meta-analysis of observational studies. Int J Cancer. 2014;134(5):1166-73.

12. Yin J, Jin X, Shan Z, Li S, Huang H, Li P, et al. Relationship of sleep duration with all-cause mortality and cardiovascular events: a systematic review and 
dose-response meta-analysis of prospective cohort studies. J Am Heart Assoc. 2017;6(9):e5947.

13. Jean-Louis G, Williams NJ, Sarpong D, Pandey A, Youngstedt S, Zizi F, et al. Associations between inadequate sleep and obesity in the US adult population: analysis of the national health interview survey (1977-2009). BMC Public Health. 2014;14(1):290.

14. Grandner MA, Patel NP, Gehrman PR, Perlis ML, Pack Al. Problems associated with short sleep: bridging the gap between laboratory and epidemiological studies. Sleep Med Rev. 2010;14(4):239-47.

15. Young T. Increasing sleep duration for a healthier (and less obese?) population tomorrow. Sleep. 2008;31(5):593-4.

16. Heckman CJ, Kloss JD, Feskanich D, Culnan E, Schernhammer ES. Associations among rotating night shift work, sleep and skin cancer in Nurses' health study II participants. Occup Environ Med. 2017;74(3):169-75.

17. Gu F, Xiao Q, Chu LW, Yu K, Matthews CE, Hsing AW, et al. Sleep duration and cancer in the NIH-AARP diet and health study cohort. PLOS One. 2016;11(9):e161561.

18. Markt SC, Flynn-Evans EE, Valdimarsdottir UA, Sigurdardottir LG, Tamimi RM, Batista JL, et al. Sleep duration and disruption and prostate cancer risk: a 23-year prospective study. Cancer Epidemiol Biomark Prev. 2016;25(2):302-8.

19. Luojus MK, Lehto SM, Tolmunen T, Erkkilä AT, Kauhanen J. Sleep duration and incidence of lung cancer in ageing men. BMC Public Health. 2014;14(1):295.

20. Jiao L, Duan Z, Sangi-Haghpeykar H, Hale L, White DL, El-Serag HB. Sleep duration and incidence of colorectal cancer in postmenopausal women. $\mathrm{Br}$ J Cancer. 2013;108(1):213-21.

21. Zhang X, Giovannucci EL, Wu K, Gao X, Hu F, Ogino S, et al. Associations of self-reported sleep duration and snoring with colorectal cancer risk in men and women. Sleep. 2013;36(5):681-8.

22. Xiao Q, Signorello LB, Brinton LA, Cohen SS, Blot WJ, Matthews CE. Sleep duration and breast cancer risk among black and white women. Sleep Med. 2016;20:25-9.

23. Cohen JM, Li YT, Wu S, Han J, Qureshi AA, Cho E. Sleep duration and sleepdisordered breathing and the risk of melanoma among US women and men. Int J Dermatol. 2015;54(11):e492-5.

24. Hurley S, Goldberg D, Bernstein L, Reynolds P. Sleep duration and cancer risk in women. Cancer Causes Control. 2015;26(7):1037-45.

25. Moher D, Liberati A, Tetzlaff J, Altman DG. Preferred reporting items for systematic reviews and meta-analyses: the PRISMA statement. BMJ. 2009;339:b2535.

26. Liberati A, Altman DG, Tetzlaff J, Mulrow C, Gotzsche PC, loannidis JP, et al. The PRISMA statement for reporting systematic reviews and meta-analyses of studies that evaluate healthcare interventions: explanation and elaboration. BMJ. 2009;339:b2700.

27. DerSimonian R, Laird N. Meta-analysis in clinical trials. Control Clin Trials. 1986;7(3):177-88.

28. Higgins JPT, Green S (editors). (2011). Cochrane Handbook for Systematic Reviews of Interventions Version 5.1.0 [updated March 2011]. The Cochrane Collaboration, 2011. Available from http://handbook.cochrane.org.

29. McElroy JA, Newcomb PA, Titus-Ernstoff L, Trentham-Dietz A, Hampton JM, Egan KM. Duration of sleep and breast cancer risk in a large populationbased case-control study. J Sleep Res. 2006;15(3):241-9.

30. Markt SC, Grotta A, Nyren O, Adami H, Mucci LA, Valdimarsdottir UA, et al. Insufficient sleep and risk of prostate cancer in a large Swedish cohort. Sleep. 2015;38(9):1405-10.

31. Vogtmann E, Levitan EB, Hale L, Shikany JM, Shah NA, Endeshaw Y, et al. Association between sleep and breast cancer incidence among postmenopausal women in the Women's Health Initiative. Sleep. 2013; 36(10):1437-44.

32. Pinheiro SP, Schernhammer ES, Tworoger SS, Michels KB. A prospective study on habitual duration of sleep and incidence of breast cancer in a large cohort of women. Cancer Res. 2006;66(10):5521-5.

33. Qian X, Brinton LA, Schairer C, Matthews CE. Sleep duration and breast cancer risk in the breast cancer detection demonstration project follow-up cohort. Br J Cancer. 2015;112(3):567-71.

34. von Ruesten A, Weikert C, Fietze I, Boeing H. Association of Sleep Duration with chronic diseases in the European prospective investigation into cancer and nutrition (EPIC)-Potsdam study. PLoS One. 2012;7(1):e30972.

35. Weiderpass E, Sandin S, Inoue M, Shimazu T, Iwasaki M, Sasazuki S, et al. Risk factors for epithelial ovarian cancer in Japan - results from the Japan public health center-based prospective study cohort. Int J Oncol. 2012;40(1):21-30.
36. Girschik J, Heyworth J, Fritschi L. Self-reported sleep duration, sleep quality, and breast cancer risk in a population-based case-control study. Am J Epidemiol. 2013;177(4):316-27.

37. Khawaja O, Petrone AB, Aleem S, Manzoor K, Gaziano JM, Djousse L. Sleep duration and risk of lung cancer in the physicians' health study. Zhongguo Fei Ai Za Zhi. 2014;17(9):649-55.

38. Luo J, Sands M, Wactawski-Wende J, Song Y, Margolis KL. Sleep disturbance and incidence of thyroid cancer in postmenopausal women the Women's Health Initiative. Am J Epidemiol. 2013;177(1):42-9.

39. Sturgeon SR, Luisi N, Balasubramanian R, Reeves KW. Sleep duration and endometrial cancer risk. Cancer Causes Control. 2012;23(4):547-53.

40. Kakizaki M, Inoue K, Kuriyama S, Sone T, Matsuda-Ohmori K, Nakaya N, et al. Sleep duration and the risk of prostate cancer: the Ohsaki cohort study. Br J Cancer. 2008;99(1):176-8.

41. Kakizaki M, Kuriyama S, Sone T, Ohmori-Matsuda K, Hozawa A, Nakaya N, et al. Sleep duration and the risk of breast cancer: the Ohsaki cohort study. $\mathrm{Br}$ J Cancer. 2008;99(9):1502-5.

42. Verkasalo PK, Lillberg K, Stevens RG, Hublin C, Partinen M, Koskenvuo M, et al. Sleep duration and breast cancer: a prospective cohort study. Cancer Res. 2005;65(20):9595-600.

43. Wang P, Ren F, Lin Y, Su F, Jia W, Su X, et al. Night-shift work, sleep duration, daytime napping, and breast cancer risk. Sleep Med. 2015;16(4):462-8.

44. Wu AH, Stanczyk FZ, Wang R, Koh W, Yuan J, Yu MC. Sleep duration, spot urinary 6-sulfatoxymelatonin levels and risk of breast cancer among Chinese women in Singapore. Int J Cancer. 2013;132(4):891-6.

45. II Yasova D, Hertz-Picciotto I, Peters U, Berlin JA, Poole C. Choice of exposure scores for categorical regression in meta-analysis: a case study of a common problem. Cancer Causes Control. 2005;16(4):383-8.

46. Hartemink N, Boshuizen HC, Nagelkerke NJD, Jacobs MAM, van Houwelingen HC. Combining risk estimates from observational studies with different exposure Cutpoints: a meta-analysis on body mass index and diabetes type 2. Am J Epidemiol. 2006;163(11):1042-52.

47. Xu C, Kwong JSW, Zhang C. The use of piecewise linear spline function on dose-response meta-analysis. Ann Transl Med. 2016;4(19):389.

48. Higgins JPT, Thompson SG. Quantifying heterogeneity in a meta-analysis. Stat Med. 2002;21(11):1539-58.

49. Higgins JPT. Measuring inconsistency in meta-analyses. BMJ. 2003;327(7414): 557-60.

50. Brown SB, Hankinson SE. Endogenous estrogens and the risk of breast, endometrial, and ovarian cancers. Steroids. 2015;99(Pt A):8-10.

51. Takayama Kl, Misawa A, Inoue S. Significance of microRNAs in androgen signaling and prostate cancer progression. Cancers (Basel). 2017;9(8).

52. Egger M, Davey SG, Schneider M, Minder C. Bias in meta-analysis detected by a simple, graphical test. BMJ. 1997;315(7109):629-34.

53. Begg CB, Mazumdar M. Operating characteristics of a rank correlation test for publication bias. Biometrics. 1994;50(4):1088-101.

54. Peters JL, Sutton AJ, Jones DR, Abrams KR, Rushton L. Contour-enhanced meta-analysis funnel plots help distinguish publication bias from other causes of asymmetry. J Clin Epidemiol. 2008;61(10):991-6.

55. Wu AH, Wang R, Koh WP, Stanczyk FZ, Lee HP, Yu MC. Sleep duration, melatonin and breast cancer among Chinese women in Singapore. Carcinogenesis. 2008;29(6):1244-8.

56. Devore EE, Massa J, Papantoniou K, Schernhammer ES, Wu K, Zhang X, et al. Rotating night shift work, sleep, and colorectal adenoma in women. Int J Color Dis. 2017:32(7):1013-8.

57. Thompson CL, Larkin EK, Patel S, Berger NA, Redline S, Li L. Short duration of sleep increases risk of colorectal adenoma. Cancer. 2011;117(4):841-7.

58. Moher D, Liberati A, Tetzlaff J, Altman DG. Preferred reporting items for systematic reviews and meta-analyses: the PRISMA statement. Ann Intern Med. 2009;151(4):264-9 W64.

59. Erren TC, Morfeld P, Foster RG, Reiter RJ, Groß JV, Westermann IK. Sleep and cancer: synthesis of experimental data and meta-analyses of cancer incidence among some 1,500,000 study individuals in 13 countries. Chronobiol Int. 2016;33(4):325-50.

60. Lu Y, Tian N, Yin J, Shi Y, Huang Z. Association between sleep duration and cancer risk: a meta-analysis of prospective cohort studies. PLoS One. 2013;8(9):e74723.

61. Zhao H, Yin JY, Yang WS, Qin Q, Li TT, Shi Y, et al. Sleep duration and cancer risk: a systematic review and meta-analysis of prospective studies. Asian Pac J Cancer Prev. 2013;14(12):7509-15. 
62. Fritschi L, Glass DC, Heyworth JS, Aronson K, Girschik J, Boyle T, et al. Hypotheses for mechanisms linking shiftwork and cancer. Med Hypotheses. 2011;77(3):430-6.

63. Blask DE. Melatonin, sleep disturbance and cancer risk. Sleep Med Rev. 2009;13(4):257-64.

64. Yao PL, Gonzalez FJ, Peters JM. Targeting estrogen receptor-beta for the prevention of nonmelanoma skin cancer. Cancer Prev Res (Phila). 2014;7(2):182-5

65. de Giorgi V, Gori A, Alfaioli B, Papi F, Grazzini M, Rossari S, et al. Influence of sex hormones on melanoma. J Clin Oncol. 2011;29(4):e94-5 e96.

66. Folkerd EJ, Dowsett M. Influence of sex hormones on cancer progression. J Clin Oncol. 2010;28(26):4038-44.

67. Shang Y. Hormones and cancer. Cell Res. 2007;17(4):277-9.

68. Derwahl M, Nicula D. Estrogen and its role in thyroid cancer. Endocr Relat Cancer. 2014:21(5):T273-83.

69. Liang J, Shang Y. Estrogen and cancer. Annu Rev Physiol. 2013;75:225-40.

70. Yang W, Shi Y, Ke X, Sun H, Guo J, Wang X. Long-term sleep habits and the risk of breast cancer among Chinese women: a case-control study. Eur J Cancer Prev. 2018.

71. Cos S, Gonzalez A, Martinez-Campa C, Mediavilla MD, Alonso-Gonzalez C, Sanchez-Barcelo EJ. Melatonin as a selective estrogen enzyme modulator. Curr Cancer Drug Targets. 2008;8(8):691-702.

72. Alvarez-Garcia V, Gonzalez A, Martinez-Campa C, Alonso-Gonzalez C, Cos S. Melatonin modulates aromatase activity and expression in endothelial cells. Oncol Rep. 2013;29(5):2058-64.

73. Yang WS, Deng Q, Fan WY, Wang WY, Wang X. Light exposure at night, sleep duration, melatonin, and breast cancer: a dose-response analysis of observational studies. Eur J Cancer Prev. 2014;23(4):269-76.

74. Schernhammer ES, Rosner B, Willett WC, Laden F, Colditz GA, Hankinson SE. Epidemiology of urinary melatonin in women and its relation to other hormones and night work. Cancer Epidemiol Biomark Prev. 2004;13(6):936-43.

75. Okatani Y, Morioka N, Wakatsuki A. Changes in nocturnal melatonin secretion in perimenopausal women: correlation with endogenous estrogen concentrations. J Pineal Res. 2000;28(2):111-8.

76. Clark ML, Burch JB, Yost MG, Zhai Y, Bachand AM, Fitzpatrick CT, et al. Biomonitoring of estrogen and melatonin metabolites among women residing near radio and television broadcasting transmitters. J Occup Environ Med. 2007:49(10):1149-56.

77. Bovbjerg DH. Circadian disruption and cancer: sleep and immune regulation. Brain Behav Immun. 2003;17(Suppl 1):S48-50.

78. Bollinger $\mathrm{T}$, Bollinger $\mathrm{A}$, Oster $\mathrm{H}$, Solbach W. Sleep, immunity, and circadian clocks: a mechanistic model. Gerontology. 2010;56(6):574-80.

79. Patel SR, Zhu X, Storfer-Isser A, Mehra R, Jenny NS, Tracy R, et al. Sleep duration and biomarkers of inflammation. Sleep. 2009;32(2):200-4.

80. Konturek PC, Brzozowski T, Konturek SJ. Gut clock: implication of circadian rhythms in the gastrointestinal tract. J Physiol Pharmacol. 2011;62(2):139-50.

81. Knutson KL, Spiegel K, Penev P, Van Cauter E. The metabolic consequences of sleep deprivation. Sleep Med Rev. 2007;11(3):163-78.

82. Antonova L, Aronson K, Mueller CR. Stress and breast cancer: from epidemiology to molecular biology. Breast Cancer Res. 2011;13(2):208

83. Lochan R, Daly AK, Reeves HL, Charnley RM. Family history of cancer and tobacco exposure in index cases of pancreatic ductal adenocarcinoma. J Oncol. 2011:2011:215985.

84. Percik R, Stumvoll M. Obesity and cancer. Exp Clin Endocrinol Diabetes. 2009:117(10):563-6.

85. Knutson KL. Does inadequate sleep play a role in vulnerability to obesity? Am J Hum Biol. 2012;24(3):361-71.

86. Joranby L. PKGM. Addiction to food and brain reward systems. Sex Addict Compulsivity. 2005;12(2-3):201-17.

87. Mokdad AH, Ford ES, Bowman BA, Dietz WH, Vinicor F, Bales VS, et al. Prevalence of obesity, diabetes, and obesity-related health risk factors, 2001. JAMA. 2003;289(1):76-9.

88. Must A, Spadano J, Coakley EH, Field AE, Colditz G, Dietz WH. The disease burden associated with overweight and obesity. JAMA. 1999;282(16):1523-9.

89. Knutson KL, Turek FW. The U-shaped association between sleep and health: the 2 peaks do not mean the same thing. Sleep. 2006;29(7):878-9.

90. Stranges S, Dorn JM, Shipley MJ, Kandala NB, Trevisan M, Miller MA, et al. Correlates of short and long sleep duration: a cross-cultural comparison between the United Kingdom and the United States: the Whitehall II study and the Western New York health study. Am J Epidemiol. 2008;168(12):1353-64.
91. Girschik J, Heyworth J, Fritschi L. Re: "night-shift work and breast cancer risk in a cohort of Chinese women". Am J Epidemiol. 2010;172(7):865-6 867-68.

92. Wetterberg L, Halberg F, Tarquini B, Cagnoni M, Haus E, Griffith K, et al. Circadian variation in urinary melatonin in clinically healthy women in Japan and the United States of America. Experientia. 1979;35(3):416-9.

93. Wetterberg L, Halberg F, Halberg E, Haus E, Kawasaki T, Ueno M, et al. Circadian characteristics of urinary melatonin from clinically healthy young women at different civilization disease risks. Acta Med Scand. 1986:220(1):71-81.

94. Budhrani PH, Lengacher CA, Kip KE, Tofthagen C, Jim H. Minority breast cancer survivors: the association between race/ethnicity, objective sleep disturbances, and physical and psychological symptoms. Nurs Res Pract. 2014:2014:858403.

95. Zheng B, Lin LL, Yu CQ, Lyu J, Guo Y, Bian Z, et al. Distributions and associations between duration of sleep, daytime naps and insomnia symptoms among Chinese adults. Zhonghua Liu Xing Bing Xue Za Zhi. 2017;38(4):452-6.

96. Ohayon MM, Milesi C. Artificial outdoor nighttime lights associate with altered sleep behavior in the American general population. Sleep. 2016:39(6):1311-20.
Ready to submit your research? Choose BMC and benefit from:

- fast, convenient online submission

- thorough peer review by experienced researchers in your field

- rapid publication on acceptance

- support for research data, including large and complex data types

- gold Open Access which fosters wider collaboration and increased citations

- maximum visibility for your research: over $100 \mathrm{M}$ website views per year

At $\mathrm{BMC}$, research is always in progress.

Learn more biomedcentral.com/submissions 